\title{
An Easy Completeness Proof for the Modal $\mu$-Calculus on Finite Trees
}

\author{
Balder ten Cate $^{1, *}$ and Gaëlle Fontaine ${ }^{2, \star}$ \\ 1 University of California, Santa Cruz \\ 2 ILLC, Universiteit van Amsterdam
}

\begin{abstract}
We give a complete axiomatization for the modal $\mu$-calculus on finite trees. While the completeness of our axiomatization already follows from a more powerful result by Igor Walukiewicz in [11, our proof is easier and uses very different tools, inspired from model theory. We show that our approach generalizes to certain axiomatic extensions, and to the extension of the $\mu$-calculus with graded modalities. We hope that the method might be helpful for other completeness proofs as well.
\end{abstract}

The $\mu$-calculus is an extension of modal logic with a fixpoint operator. In 1983, Dexter Kozen suggested an axiomatization and showed completeness for the aconjunctive fragment of the $\mu$-calculus (see, e.g., 7]). It took more than ten years to prove completeness. This proof is due to Igor Walukiewicz [11] and is quite involved. It uses tableaux and the notion of disjunctive formula. We propose here a simpler proof in a particular case. More precisely, we prove the completeness of the Kozen axiomatization $\mathbf{K}^{\mu}$ extended with the axiom $\mu x . \square x$ with respect to the class of finite tree models. Finite trees are a fundamental data structure in computer science, and logics on finite trees have received considerable attention in recent literature, motivated by applications in areas such as XML 18 .

Our argument consists of three steps. The first step consists of defining a notion of rank which plays the same role as the modal depth for modal formulas. One of the main properties of the rank is the following. In order to know whether a formula $\varphi$ of rank $n$ is true at a node $w$, it is enough to know which proposition letters are true at $w$ and which formulas of rank at most $n$ are true at the successor nodes of $w$. Another key property of the rank is that there are only finitely many formulas of a given rank (up to logical equivalence).

The second step is to prove completeness of the $\mu$-calculus with respect to generalized models, which are basically Kripke models augmented with a set of admissible subsets, in the style of Henkin semantics for second order logic.

\footnotetext{
* The first author was supported by the Netherlands Organization for Scientific Research (NWO), under grant 639.021.508 and by ERC Advanced Grand Webdam on Foundation of Web data management. The second author is supported by VICI grant 639.073.501 of the NWO. We are grateful to Alexandru Baltag and Yde Venema for their comments on earlier drafts. We also thank the anonymous referees for helpful remarks.
} 
The last step is inspired by the work of Kees Doets (see, e.g., [3]). Let us call a node in a generalized model $n$-good if there is a node in a finite tree model which satisfies exactly the same formulas of rank at most $n$. Using an induction principle, we show that every node in a generalized model satisfying $\mu x . \square x$ is $n$-good. It is here that we use the main property of the rank. Finally, putting this together with the completeness for generalized models, we obtain completeness for the class of finite tree models.

This argument can also be applied to some extensions of the logic $\mathbf{K}^{\mu}+\mu x . \square x$. More precisely, we prove that when we add finitely many shallow axioms (as defined in [10]), we obtain a complete axiomatization for the corresponding class of finite trees. We also show that we can adapt our proof to show completeness for the graded $\mu$-calculus extended with the axiom $\mu x . \square x$. Let us also mention that a similar method has been used for other completeness proofs in [6].

The paper is organized as follows. In section 1, we recall what is the Kozen axiomatization for the $\mu$-calculus $\mathbf{K}^{\mu}$ and what is the intended semantics. In section 2 , we define the notion of rank for a formula. In section 3 , we give a definition for the generalized models and we show completeness of $\mathbf{K}^{\mu}$ with respect to the class of generalized models. In section 4, we use Kees Doets' argument to obtain completeness of $\mathbf{K}^{\mu}+\mu x . \square x$ with respect to the class of finite tree models. In the last two sections, we give some examples of extensions of $\mathbf{K}^{\mu}+\mu x$. $\square x$ to which we can apply our method in order to prove completeness.

\section{Syntax, Semantics and Axiomatization}

We introduce the language and the Kripke semantics for the $\mu$-calculus. We also recall the axiomatization given by Dexter Kozen.

Definition 1. The $\mu$-formulas over a set Prop of proposition letters and a set Var of variables are given by

$$
\varphi::=\top|p| x|\varphi \vee \varphi| \neg \varphi|\nabla \varphi| \mu x \cdot \varphi,
$$

where $p$ ranges over the set Prop and $x$ ranges over the set $V$ ar of variables. In $\mu x . \varphi$, we require that the variable $x$ appears only under an even number of negations in $\varphi$. We will assume that $\operatorname{Var}$ is infinite.

As usual, we let $\phi \wedge \psi, \square \varphi$ and $\nu x . \varphi$ be abbreviations for $\neg(\neg \varphi \vee \neg \psi), \neg \diamond \neg \varphi$ and $\neg \mu x . \neg[\neg x / x]$. The notions of subformula, bound variable, free variable and substitution are defined in the usual way. If $\varphi$ and $\psi$ are $\mu$-formulas and if $p$ is a proposition letter, we denote by $\varphi[\psi / p]$ the formula obtained by replacing in $\varphi$ each occurrence of $p$ by $\psi$. Similarly, if $x$ is a variable, we define $\varphi[\psi / x]$.

A $\mu$-sentence is a formula in which all the variables are bound. A $\mu$-formula is a modal formula if it does not contain any subformula of the form $\mu x . \varphi$.

Definition 2. A Kripke frame is a pair $(W, R)$, where $W$ is a set and $R$ a binary relation on $W$. A Kripke model is a triple $(W, R, V)$ where $(W, R)$ is a Kripke frame and $V:$ Prop $\rightarrow \mathcal{P}(W)$ a valuation. If $(w, v)$ belongs to $R$, we say that $w$ is a predecessor of $v$ and $v$ is a successor of $w$. 
Given a formula $\varphi$, a Kripke model $\mathcal{M}=(W, R, V)$ and an assignment $\tau:$ Var $\rightarrow$ $\mathcal{P}(W)$, we define a subset $\llbracket \varphi \rrbracket_{\mathcal{M}, \tau}$ that is interpreted as the set of points at which $\varphi$ is true. The subset is defined by induction in the usual way. We only recall that

$$
\llbracket \mu x . \varphi \rrbracket_{\mathcal{M}, \tau}=\bigcap\left\{U \subseteq W: \llbracket \varphi \rrbracket_{\mathcal{M}, \tau[x:=U]} \subseteq U\right\},
$$

where $\tau[x:=U]$ is the assignment $\tau^{\prime}$ such that $\tau^{\prime}(x)=U$ and $\tau^{\prime}(y)=\tau(y)$, for all $y \neq x$. Observe that the set $\llbracket \mu x . \varphi \rrbracket_{\mathcal{M}, \tau}$ is the least fixpoint of the map $\varphi_{x}: \mathcal{P}(W) \rightarrow \mathcal{P}(W)$ defined by $\varphi_{x}(U):=\llbracket \varphi \rrbracket_{\mathcal{M}, \tau[x:=U]}$, for all $U \subseteq W$.

If $w \in \llbracket \varphi \rrbracket_{\mathcal{M}, \tau}$, we write $\mathcal{M}, w \Vdash_{\tau} \varphi$ and we say that $\varphi$ is true at $w$ under the assignment $\tau$. If $\varphi$ is a sentence, we simply write $\mathcal{M}, w \Vdash \varphi$.

A formula $\varphi$ is true in $\mathcal{M}$ under an assignment $\tau$ if for all $w \in W$, we have $\mathcal{M}, w \Vdash_{\tau} \varphi$. In this case, we write $\mathcal{M} \Vdash_{\tau} \varphi$. A set $\Phi$ of formulas is true in a model $\mathcal{M}$ under an assignment $\tau$, notation: $\mathcal{M} \Vdash_{\tau} \Phi$, if for all $\varphi$ in $\Phi, \varphi$ is true in $\mathcal{M}$ under $\tau$. Finally, if $(W, R)$ is a Kripke frame and for all valuations $V$ and all assignments $\tau, \varphi$ is true in $(W, R, V)$ under the assignment $\tau$, we say that $\varphi$ is valid in $(W, R)$ and we write $(W, R) \Vdash \varphi$.

Definition 3. The axiomatization of the Kozen system $\mathbf{K}^{\mu}$ consists of the following axioms and rules

$$
\begin{array}{lr}
\text { propositional tautologies, } & \\
\text { If } \vdash \varphi \rightarrow \psi \text { and } \vdash \varphi, \text { then } \vdash \psi & \text { (Modus ponens), } \\
\text { If } \vdash \varphi, \text { then } \vdash \varphi[p / \psi] & \text { (Substitution), } \\
\vdash \square(p \rightarrow q) \rightarrow(\square p \rightarrow \square q) & \text { ( } K \text {-axiom), } \\
\text { If } \vdash \varphi, \text { then } \vdash \square \varphi & \text { (Necessitation), } \\
\vdash \varphi[x / \mu x . \varphi] \rightarrow \mu x . \varphi & \text { (Fixpoint axiom), } \\
\text { If } \vdash \varphi[x / \psi] \rightarrow \psi, \text { then } \vdash \mu x . \varphi \rightarrow \psi & \text { (Fixpoint rule), }
\end{array}
$$

where $x$ is not a bound variable of $\varphi$ and no free variable of $\psi$ is bound in $\varphi$.

Definition 4. If $\Phi$ is a set of modal formulas, we write $\mathbf{K}+\Phi$ for the smallest set of modal formulas which contains the propositional tautologies, the $K$-axiom and is closed under the Modus Ponens, Substitution and Necessitation rules. We say that $\mathbf{K}+\Phi$ is the extension of $\mathbf{K}$ by $\Phi$. Note that if $\Phi$ is empty, we simply write $\mathbf{K}$.

Next, if $\Phi$ is a set of modal formulas, we denote by $\mathbf{K}+{ }_{r} \Phi$ the smallest set of formulas which contains both $\mathbf{K}$ and $\Phi$ and is closed under the Modus Ponens and Necessitation rules. We call $\mathbf{K}+{ }_{r} \Phi$ the restricted extension of $\mathbf{K}$ by $\Phi$.

Finally, if $\Phi$ is a set of $\mu$-formulas, we write $\mathbf{K}^{\mu}+\Phi$ for the smallest set of formulas which contains both $\mathbf{K}^{\mu}$ and $\Phi$ and is closed under the Modus Ponens, Substitution, Necessitation and Fixpoint rules. We say that $\mathbf{K}^{\mu}+\Phi$ is the extension of $\mathbf{K}^{\mu}$ by $\Phi$.

Definition 5. Let $(W, R)$ be a Kripke frame. A point $r$ in $W$ is a root if for all $w$ in $W$, there is a sequence $w_{0}, \ldots, w_{n}$ such that $w_{0}=r, w_{n}=w$ and $\left(w_{i}, w_{i+1}\right)$ belongs to $R$, for all $i \in\{0, \ldots, n-1\}$. The frame $(W, R)$ is a tree if it has a root, every point distinct from the root has a unique predecessor and there is no 
sequence $w_{0}, \ldots, w_{n+1}$ in $W$ such that $w_{n+1}=w_{0}$ and $\left(w_{i}, w_{i+1}\right)$ belongs to $R$, for all $i \in\{0, \ldots, n\}$.

The frame $(W, R)$ is a finite tree if it is a tree and $W$ is finite. Finally, a finite tree Kripke model is a Kripke model $(W, R, V)$ such that $(W, R)$ is a finite tree.

Proposition 1. Let $\mathcal{M}=(W, R, V)$ be a Kripke model. The formula $\mu x . \square x$ is true at a point $w$ in $\mathcal{M}$ iff there is no infinite sequence $w_{0}, w_{1} \ldots$ in $W$ such that $w_{0}=w$ and $\left(w_{i}, w_{i+1}\right)$ belongs to $R$, for all $i \in \mathbb{N}$.

In particular, the formula $\mu x . \square x$ is true in $\mathcal{M}$ iff there is no infinite sequence $w_{0}, w_{1}, \ldots$ such that $\left(w_{i}, w_{i+1}\right)$ belongs to $R$, for all $i \in \mathbb{N}$. That is, iff $\mathcal{M}$ is conversely well-founded.

We prove the completeness of the logic $\mathbf{K}^{\mu}+\mu x . \square x$ with respect to the class of finite tree Kripke models. That is, a formula $\varphi$ is provable in $\mathbf{K}^{\mu}+\mu x$. $\square x$ iff it is valid in any finite tree Kripke model. In fact, this result can be derived from the completeness result proved by Igor Walukiewicz in 11. We will give more details at the end of Section 4 .

\section{Rank of a Formula}

The goal of this section is to come up with a definition of rank that would be the analogue of the depth of a modal formula. For modal logics, it is not hard to see that the truth of an arbitrary formula $\varphi$ at some world $w$ only depends of the truth of the proposition letters at $w$ and of the truth of formulas $\psi$ at the successors of $w$, where the depth of $\psi$ is at most the depth of $\varphi$. In our proof, we will need something similar for the $\mu$-calculus.

The most natural idea would be to look at the nesting depth of modal and fixpoint operators. However, this definition does not have the required properties. The notion of rank that we develop here is in fact related to the closure of a formula, which has been introduced by Michael Fischer and Robert Ladner in [5].

Definition 6. The closure $C l(\varphi)$ of a formula $\varphi$ is the smallest set of formulas such that

$$
\begin{array}{l|l}
\varphi \in C l(\varphi), & \text { if } \psi \vee \chi \in C l(\varphi), \text { then }\{\psi, \chi\} \subseteq C l(\varphi), \\
\text { if } \neg \psi \in C l(\varphi), \text { then } \psi \in C l(\varphi), & \text { if } \mu x . \psi \in C l(\varphi), \text { then } \psi[x / \mu x . \psi] \in C l(\varphi) . \\
\text { if } \nabla \psi \in C l(\varphi), \text { then } \psi \in C l(\varphi), &
\end{array}
$$

It is also proved in [7] that the closure $C l(\varphi)$ of a formula $\varphi$ is finite. In order to define the rank, we also need to recall the notion of the depth of a formula.

Definition 7. The depth $d(\varphi)$ of a formula $\varphi$ is defined by induction as follows

$$
\begin{array}{l|l}
d(\top)=d(p)=d(x)=0, & d(\varphi \vee \psi)=\max \{d(\varphi), d(\psi)\}, \\
d(\neg \varphi)=d(\varphi), & d(\nabla \varphi)=d(\mu x . \varphi)=d(\varphi)+1
\end{array}
$$

Definition 8. The rank of a formula $\varphi$ is defined as follows

$$
\operatorname{rank}(\varphi)=\max \{d(\psi) \mid \psi \in C l(\varphi)\}
$$


Remark that since $C l(\varphi)$ is finite, $\operatorname{rank}(\varphi)$ is always a natural number. All we will use later are the following properties of the rank.

Proposition 2. If the set Prop of proposition letters is finite, then for all natural numbers $k$, there are only finitely many sentences of rank $k$ (up to logical equivalence).

Proof. Fix a natural number $k$. Note first that if $\operatorname{rank}(\varphi)=k$, then in particular, $d(\varphi) \leq k$. Hence, it is enough to show that there only finitely many sentences of depth below $k$ (up to logical equivalence). If $d(\varphi) \leq k$, we may assume that the only variables occurring in $\varphi$ are some $x_{1}, \ldots, x_{k}$. It is routine to prove by induction on $l$ that there are finitely many formulas of depth $l$ with variables $x_{1}, \ldots, x_{k}$, up to logical equivalence.

Proposition 3. The rank is closed under boolean combination. That is, for any $n$, a boolean combination of formulas of rank at most $n$ is a formula of rank at most $n$.

Proposition 4. Every formula $\varphi$ is provably equivalent to a boolean combination of proposition letters and formulas of the form $\diamond \psi$, with $\operatorname{rank}(\psi) \leq \operatorname{rank}(\varphi)$.

Proof. A formula is guarded if every bound variable is in the scope of a modal operator. Each formula $\varphi$ is provably equivalent to a guarded formula of rank less or equal to the rank of $\varphi$ (see, e.g., [1]). Therefore, let $\varphi$ be a guarded formula. We define a map $G$ by induction as follows:

$$
\begin{array}{l|l}
G(\top)=\top, & G(p)=p, \text { if } p \text { is a free variable of } \varphi, \\
G(\neg \psi)=\neg G(\psi), & G\left(\psi \vee \psi^{\prime}\right)=G(\psi) \vee G\left(\psi^{\prime}\right), \\
G(\diamond \psi)=\diamond \psi, & G(\mu x . \psi)=G(\psi[x / \mu x . \psi]) .
\end{array}
$$

We note that $G$ is not defined for a bound variable $x$ of $\varphi$. Using the fact that $\varphi$ is guarded, one can show that the computation of $G(\varphi)$ is well-defined and does terminate. It is not hard to see that $G(\varphi)$ is equivalent to $\varphi$. We remark now that if $\psi$ belongs to $C l(\varphi)$, then $C l(\psi)$ is a subset of $C l(\varphi)$. It follows that $G(\varphi)$ is a boolean combination of proposition letters and formulas of the form $\diamond \psi$, with $\operatorname{rank}(\psi) \leq \operatorname{rank}(\varphi)$.

\section{Completeness for Generalized Models}

We introduce generalized models which are the analogue for the $\mu$-calculus of the general models for second order logic. We prove completeness of $\mathbf{K}^{\mu}$ with respect to the class of generalized models.

Definition 9. Consider a quadruple $\mathcal{M}=(W, R, V, \mathbb{A})$ where $(W, R)$ is a Kripke frame, $\mathbb{A}$ is a subset of $\mathcal{P}(W)$ and $V: \operatorname{Prop} \rightarrow \mathbb{A}$ a valuation. A set which belongs to $\mathbb{A}$ is called admissible.

We define the truth of a formula $\varphi$ under an assignment $\tau: \operatorname{Var} \rightarrow \mathbb{A}$ by induction. All the clauses are the same as usual, except the one defining the 
truth of $\mu x . \varphi$. Normally, we define the set $\llbracket \mu x . \varphi \rrbracket_{\mathcal{M}, \tau}$ as the least pre-fixpoint of the map $\varphi_{x}$ (see Definition 2). But here, we define it as the intersection of all the admissible pre-fixpoints of $\varphi_{x}$.

$$
\begin{aligned}
& \llbracket \top \rrbracket_{\mathcal{M}, \tau}=W, \\
& \llbracket p \rrbracket_{\mathcal{M}, \tau}=V(p), \\
& \llbracket x \rrbracket_{\mathcal{M}, \tau}=\tau(x), \\
& \llbracket \neg \varphi \rrbracket_{\mathcal{M}, \tau}=W \backslash \llbracket \varphi \rrbracket_{\mathcal{M}, \tau}, \\
& \llbracket \varphi \vee \psi \rrbracket_{\mathcal{M}, \tau}=\llbracket \varphi \rrbracket_{\mathcal{M}, \tau} \cup \llbracket \psi \rrbracket_{\mathcal{M}, \tau}, \\
& \llbracket \diamond \varphi \rrbracket_{\mathcal{M}, \tau}=\left\{w \in W: \exists v \in W \text { s.t. } w R v \text { and } v \in \llbracket \varphi \rrbracket_{\mathcal{M}, \tau}\right\}, \\
& \llbracket \mu x . \varphi \rrbracket_{\mathcal{M}, \tau}=\bigcap\left\{U \in \mathbb{A}: \llbracket \varphi \rrbracket_{\mathcal{M}, \tau[x:=U] \subseteq U\},}\right.
\end{aligned}
$$

where $\tau[x:=U]$ is the assignment $\tau^{\prime}$ such that $\tau^{\prime}(x)=U$ and $\tau(y)=\tau(y)$, for all $y \neq x$. If $w \in \llbracket \varphi \rrbracket_{\mathcal{M}, \tau}$, we write $\mathcal{M}, w \Vdash_{\tau} \varphi$ and we say that $\varphi$ is true at $w$ under the assignment $\tau$. If $\varphi$ is a sentence, we simply write $\mathcal{M}, w \Vdash \varphi$. A formula $\varphi$ is true in $\mathcal{M}$ under an assignment $\tau$ if for all $w \in W$, we have $\mathcal{M}, w \Vdash_{\tau} \varphi$. In this case, we write $\mathcal{M} \Vdash_{\tau} \varphi$.

The quadruple $\mathcal{M}=(W, R, V, \mathbb{A})$ is a generalized model if for all formulas $\varphi$ and all assignments $\tau: \operatorname{Var} \rightarrow \mathbb{A}$, the set $\llbracket \varphi \rrbracket_{\mathcal{M}, \tau}$ belongs to $\mathbb{A}$. A triple $\mathcal{F}=(W, R, \mathcal{A})$ is a generalized frame if for every valuation $V:$ Prop $\rightarrow \mathbb{A}$, the quadruple $(W, R, V, \mathbb{A})$ is a generalized model.

If $\mathcal{F}=(W, R, \mathcal{A})$ is a generalized frame, we call $(W, R)$ the underlying Kripke frame of $\mathcal{F}$. A formula $\varphi$ is valid in a generalized frame $\mathcal{F}=(W, R, \mathcal{A})$, notation: $\mathcal{F} \Vdash \varphi$, if for all valuations $V: \operatorname{Prop} \rightarrow \mathbb{A}$ and all assignments $\tau: \operatorname{Var} \rightarrow \mathbb{A}$, the formula $\varphi$ is true in $(W, R, V, \mathbb{A})$ under the assignment $\tau$.

Any Kripke model $M=(W, R, V)$ can be seen as the generalized model $M^{\prime}=$ $(W, R, V, \mathcal{P}(W))$. It follows easily from our definition that for all formulas $\varphi$ and all points $w \in W$,

$$
M, w \Vdash \varphi \quad \text { iff } \quad M^{\prime}, w \Vdash \varphi .
$$

Now we show that for all sets $\Phi$ of formulas, the logic $\mathbf{K}^{\mu}+\Phi$ is complete with respect to a particular generalized model. If we were only interested in showing that $\mathbf{K}^{\mu}+\mu x . \square x$ is complete, we would restrict ourselves to prove that $\mathbf{K}^{\mu}+\mu x . \square x$ is complete with respect to a particular generalized model. But later, we will also show completeness for some extensions of $\mathbf{K}^{\mu}$ and it will become handy to suppose that $\Phi$ contains other additional axioms.

First we introduce some definitions and recall some results of modal logic.

Theorem 1 ([9]). Let $\Phi$ be a set of modal formulas. There exists a model $\mathcal{M}$ such that for all modal formulas $\varphi, \varphi$ is provable in $\mathbf{K}+{ }_{r} \Phi$ iff $\mathcal{M} \Vdash \varphi$.

This theorems says that every (restricted) extension of $\mathbf{K}$ is complete with respect to a class of Kripke models. However, all of these extensions might not be complete with respect to a class of Kripke frames. Indeed, there is no guarantee that the formulas in $\Phi$ are valid in the frame corresponding to the model given by Theorem 1 . 
Definition 10. Let Prop be a set of proposition letters and $\operatorname{Var}$ a set of variables. We let $\mu F L$ be the set of sentences of the form $\mu x . \varphi$ or $\nu x . \varphi$, for some $\mu$-formula $\varphi$ over Prop. We denote by $\operatorname{Prop}^{+}$the set Prop $\cup\left\{p_{\varphi}: \varphi \in \mu F L\right\}$.

If $\varphi$ is a $\mu$-formula over $\operatorname{Prop}^{+}$, we define $s(\varphi)$ as the formula obtained by replacing each proposition letter of the form $p_{\psi}(\psi \in \mu F L)$, by the formula $\psi$. We call $s(\varphi)$ the source of $\varphi$.

Next, if $\varphi$ is a $\mu$-formula over $\operatorname{Prop}^{+}$, we say that a modal formula $\psi$ over Prop $^{+}$is the replacement of $\varphi$ if $\psi$ is obtained by replacing in the formula $s(\varphi)$ all maximal subformulas $\chi$ in $\mu F L$, by the proposition letter $p_{\chi}$. In this case, we use the notation $\operatorname{repl}(\varphi)$. Finally, if $\Sigma$ is a set of $\mu$-formulas over Prop, we let $\operatorname{repl}(\Sigma)$ be the set $\{\operatorname{repl}(\varphi): \varphi \in \Sigma\}$.

For example, let $\varphi$ be the formula $\diamond\left(\mu x \cdot p_{\nu y . x \wedge y}\right)$. Then $s(\varphi)$ is the formula $\diamond(\mu x . \nu y .(x \wedge y))$ and $\operatorname{repl}(\varphi)$ is the formula $\diamond p_{\mu x . \nu y .(x \wedge y)}$. We remark also that for all formulas $\psi, \operatorname{repl}(\psi)$ is a modal formula over $\operatorname{Prop}^{+}$.

Now we will prove that for all sets of formulas $\Phi$, the logic $\mathbf{K}^{\mu}+\Phi$ is complete with respect to the class of generalized models which make $\Phi$ true. An easy way to show this would be to do a standard canonical model construction (inspired by the one used for the completeness of the modal logic $\mathbf{K}$ ).

However, we give here another proof. The idea is to use the replacement map introduced previously in order to translate the completeness result for modal logic into a completeness result for generalized Kripke models. This proof might seem a bit more tedious. In fact, it will make our result easier to extend to other settings (like graded $\mu$-calculus).

Theorem 2. Let $\Phi$ be a set of $\mu$-formulas over a set Prop. There is a generalized model $\mathcal{M}=(W, R, V, \mathbb{A})$ such that for all sentences $\varphi, \varphi$ is provable in $\mathbf{K}^{\mu}+\Phi$ iff $\mathcal{M} \Vdash \varphi$.

In particular, the logic $\mathbf{K}^{\mu}+\Phi$ is complete with respect to the class of generalized models which make $\Phi$ true. That is, for all sentences $\varphi, \varphi$ is provable in $\mathbf{K}^{\mu}+\Phi$ iff for all generalized models $\mathcal{M}$ such that $\mathcal{M} \Vdash \Phi$, we have $\mathcal{M} \Vdash \varphi$.

Proof. By Theorem 1, there is a Kripke model $\mathcal{N}=\left(W, R, V^{+}\right)$(over Prop $\left.{ }^{+}\right)$ such that for all modal formulas $\alpha$ over $\operatorname{Prop}^{+}, \alpha$ is provable in $\mathbf{K}+{ }_{r} \operatorname{repl}\left(\mathbf{K}^{\mu}+\Phi\right)$ iff $\mathcal{N} \Vdash \alpha$. Now let $\mathbb{A}$ be the set $\left\{\llbracket \delta \rrbracket_{\mathcal{N}}: \delta\right.$ modal formula over Prop $\left.{ }^{+}\right\}$. We define $\mathcal{M}$ as the quadruple $\left(W, R, V^{+}, \mathbb{A}\right)$.

First, we show that for all $\mu$-formulas $\varphi$ over Prop ${ }^{+}$, all $v$ in $W$ and all assignments $\tau: \operatorname{Var} \rightarrow \mathbb{A}$, we have

$$
\mathcal{N}, v \Vdash_{\tau} \operatorname{repl}(\varphi) \quad \text { iff } \quad \mathcal{M}, v \Vdash_{\tau} \varphi .
$$

The proof is by induction on the complexity of $\psi$. We skip the details by lack of space. Next, we prove that for all $\mu$-sentences $\varphi$ (over Prop), we have

$$
\mathcal{M} \Vdash \varphi \quad \text { iff } \quad \varphi \text { is provable in } \mathbf{K}^{\mu}+\Phi .
$$

For the direction from left to right, suppose that $\varphi$ is not provable in $\mathbf{K}^{\mu}+\Phi$. This implies that $\operatorname{repl}(\varphi)$ is not provable in $\mathbf{K}+{ }_{r} \operatorname{repl}\left(\mathbf{K}^{\mu}+\Phi\right)$. Therefore, the 
formula $\operatorname{repl}(\varphi)$ is not true in $\mathcal{N}$. By equivalence (11), this means that $\varphi$ is not true in $\mathcal{M}$.

For the direction from right to left, assume that $\varphi$ is provable in $\mathbf{K}^{\mu}+\Phi$. It is routine to show that for all generalized models $\mathcal{M}^{\prime}$ such that $\mathcal{M}^{\prime} \Vdash \Phi$, we have that $\mathcal{M}^{\prime} \Vdash \varphi$. Moreover, using equivalence (1) together with the fact that $\operatorname{repl}(\Phi)$ is true in $\mathcal{N}$, we obtain that $\Phi$ is true in $\mathcal{M}$. Putting everything together, we get that $\varphi$ is true in $\mathcal{M}$.

To finish the proof, it remains to show that $\mathcal{M}$ is a generalized model. That is, for all $\mu$-formulas $\varphi$ over Prop, the set $\llbracket \varphi \rrbracket_{\mathcal{M}}$ belongs to $\mathbb{A}$. Fix a $\mu$-formula $\varphi$ over Prop. By equivalence (1), the set $\llbracket \varphi \rrbracket_{\mathcal{M}}$ is equal to $\llbracket \operatorname{repl}(\varphi) \rrbracket_{\mathcal{N}}$. By definition of $\mathbb{A}$, this set belongs to $\mathbb{A}$.

\section{Completeness for Finite Tree Models}

In the style of Kees Doets [3, we prove completeness of $\mathbf{K}^{\mu}+\mu x . \square x$ with respect to the class of finite tree Kripke models. The argument is as follows. First, we say that a point $w$ in a generalized model is $n$-good if there is a point $v$ in a finite tree Kripke model such that no formula of rank at most $n$ can distinguish $w$ from $v$. Next, we show that "being $n$-good" is a property that can be expressed by a formula $\gamma_{n}$ of rank at most $n$. Afterwards, we prove that each point (in a generalized model) satisfying $\mu x . \square x$, is $n$-good. Finally, using completeness for generalized models, we obtain completeness of $\mathbf{K}^{\mu}+\mu x . \square x$ with respect to the class of finite tree Kripke models.

In this section, we will assume that the set Prop of proposition letters is finite. Often we write "finite tree" instead of "finite tree Kripke model".

Definition 11. Fix a natural number $n$. Let $\mathcal{M}$ and $\mathcal{M}^{\prime}$ be two generalized models. A world $w \in \mathcal{M}$ is rank $n$-indistinguishable to a world $w^{\prime} \in \mathcal{M}^{\prime}$ if for all formulas $\varphi$ of rank at most $n$, we have

$$
\mathcal{M}, w \Vdash \varphi \quad \text { iff } \quad \mathcal{M}^{\prime}, w^{\prime} \Vdash \varphi \text {. }
$$

In case this happens, we write $(\mathcal{M}, w) \sim_{n}\left(\mathcal{M}^{\prime}, w^{\prime}\right)$. Finally, we say that $w \in \mathcal{M}$ is $n$-good if there exists a finite tree $\mathcal{N}$ and some $v \in \mathcal{N}$ such that $(\mathcal{M}, w) \sim_{n}$ $(\mathcal{N}, v)$.

Definition 12. Let $n$ be a natural number and let $\Phi_{n}$ be the set of formulas of rank at most $n$. For any generalized model $\mathcal{M}$ and any $w \in \mathcal{M}$, we define the $n$-type $\theta_{n}(w)$ as the set of formulas in $\Phi_{n}$ which are true at $w$.

By Proposition 2, $\Phi_{n}$ is finite (up to logical equivalence) and in particular, there are only finitely many distinct $n$-types.

Lemma 1. Let $n$ be a natural number. There exists a formula $\gamma_{n}$ of rank $n$ such that for any generalized model $\mathcal{M}$ and any $w \in \mathcal{M}$, we have

$$
\mathcal{M}, w \Vdash \gamma_{n} \quad \text { iff } \quad(\mathcal{M}, w) \text { is } n \text {-good. }
$$


Proof. Let $n$ be a natural number and let $\gamma_{n}$ be the formula defined by

$$
\gamma_{n}=\bigvee\left\{\bigwedge \theta_{n}(w) \mid w \text { is } n \text {-good }\right\},
$$

where $w$ a point in a generalized model $\mathcal{M}$ and $\bigwedge \theta_{n}(w)$ is shorthand for $\bigwedge\{\varphi$ : $\left.\varphi \in \theta_{n}(w)\right\}$. Since there are only finitely many distinct $n$-types, the formula $\gamma_{n}$ is well-defined. Moreover, from Proposition 3, it follows that the rank of $\gamma_{n}$ is $n$.

It remains to check that $\gamma_{n}$ has the required properties. It is immediate to see that if a point $w$ in a generalized model is $n$-good, then $\gamma_{n}$ is true at $w$. For the other direction, assume that $\gamma_{n}$ is true at a point $w$ in a generalized model $\mathcal{M}$. Therefore, there is a point $w^{\prime}$ in a generalized model $\mathcal{M}^{\prime}$ such that $w^{\prime}$ is $n$-good and $\theta_{n}\left(w^{\prime}\right)$ is true at $w$. Since $w^{\prime}$ is $n$-good, there is a point $v$ in a model $\mathcal{N}$ such that $w^{\prime}$ and $v$ are rank $n$-indistinguishable. Using the fact that $w$ and $w^{\prime}$ have the same $n$-type, we obtain that $w$ and $v$ are also rank $n$-indistinguishable. That is, $w$ is $n$-good.

Lemma 2. For all natural numbers $n, \vdash_{\mathbf{K}^{\mu}} \square \gamma_{n} \rightarrow \gamma_{n}$.

Proof. Let $n$ be a natural number. By Theorem 2, it is enough to show that the formula $\square \gamma_{n} \rightarrow \gamma_{n}$ is valid in all generalized models. Let $\mathcal{M}$ be a generalized model and let $w \in \mathcal{M}$. We have to show $\mathcal{M}, w \Vdash \square \gamma_{n} \rightarrow \gamma_{n}$. So suppose $\mathcal{M}, w \Vdash \square \gamma_{n}$. If $w$ is a reflexive point, we immediately obtain $\mathcal{M}, w \Vdash \gamma_{n}$ and this finishes the proof. Assume now that $w$ is irreflexive. We have to prove that $(\mathcal{M}, w)$ is $n$-good. That is, we have to find a finite tree $\mathcal{N}$ and some $v \in \mathcal{M}$ such that $(\mathcal{M}, w) \sim_{n}(\mathcal{N}, v)$.

Now for any successor $u$ of $w$, we have $\mathcal{M}, u \Vdash \gamma_{n}$. Therefore, $(\mathcal{M}, u)$ is $n$-good and there exists a finite tree $\mathcal{M}_{u}=\left(W_{u}, R_{u}, V_{u}\right)$ and some $w_{u} \in W_{u}$ such that $(\mathcal{M}, u) \sim_{n}\left(\mathcal{M}_{u}, w_{u}\right)$. Without loss of generality, we may assume that $w_{u}$ is the root of $\mathcal{M}_{u}$.

The idea is now to look at the disjoint union of these models and to add a root $v$ (that would be rank $n$-indistinguishable from $w$ ). However, this new model might not be a finite tree ( $w$ might have infinitely many successors). The solution is to restrict ourselves to finitely many successors of $w$. More precisely, for each $n$-type, we pick at most one successor of $w$.

So let $U$ be a set of successors of $w$ such that for any successor $u$ of $w$, there is exactly one point $u^{\prime}$ of $U$ satisfying $\theta_{n}(u)=\theta_{n}\left(u^{\prime}\right)$. Remark that since there are only finitely many distinct $n$-types, $U$ is finite. Let $\mathcal{N}=(W, R, V)$ be the model defined by

$$
\begin{aligned}
W & =\{v\} \cup \biguplus\left\{W_{u}: u \in U\right\}, \\
R & =\left\{\left(v, w_{u}\right): u \in U\right\} \cup \bigcup\left\{R_{u}: u \in U\right\}, \\
V(p) & = \begin{cases}\{v\} \cup \bigcup\left\{V_{u}(p): u \in U\right\} & \text { if } \mathcal{M}, w \Vdash p, \\
\bigcup\left\{V_{u}(p): u \in U\right\} & \text { otherwise, }\end{cases}
\end{aligned}
$$

for all proposition letters $p$. Since $U$ is finite, $\mathcal{N}$ is a finite tree. Thus, it is enough to check that for any formula $\varphi$ of rank at most $n$, we have

$$
\mathcal{M}, w \Vdash \varphi \quad \text { iff } \quad \mathcal{N}, v \Vdash \varphi .
$$


By Proposition 4, $\varphi$ is provably equivalent to a boolean combination of proposition letters and formulas of the form $\diamond \psi$, where $\operatorname{rank}(\psi)$ is at most $n$. Thus, it is enough to show that $w$ and $v$ satisfy exactly the same proposition letters and the same formulas $\diamond \psi$ with $\operatorname{rank}(\psi) \leq n$.

By definition of $V$, it is immediate that $w$ and $v$ satisfy the same proposition letters. Now let $\psi$ be a formula of rank at most $n$. We have to show that

$$
\mathcal{M}, w \Vdash \diamond \psi \quad \text { iff } \quad \mathcal{N}, v \Vdash \diamond \psi .
$$

For the direction from left to right, suppose that $\mathcal{M}, w \Vdash \diamond \psi$. Thus, there exists a successor $u$ of $w$ such that $\mathcal{M}, u \Vdash \psi$. By definition of $U$, there is $u^{\prime} \in U$ such that $(\mathcal{M}, u) \sim_{n}\left(\mathcal{M}, u^{\prime}\right)$. Thus, $(\mathcal{M}, u) \sim_{n}\left(\mathcal{M}_{u^{\prime}}, w_{u^{\prime}}\right)$ and in particular, $\mathcal{M}_{u^{\prime}}, w_{u^{\prime}} \Vdash \psi$. By definition of $R$, it follows that $\mathcal{N}, v \Vdash \diamond \psi$. The direction from right to left is similar.

Proposition 5. For all natural numbers $n, \vdash_{\mathbf{K}^{\mu}} \mu x . \square x \rightarrow \gamma_{n}$.

Proof. By Lemma 2, we know that $\square \gamma_{n} \rightarrow \gamma_{n}$ is provable in $\mathbf{K}^{\mu}$. By the Fixpoint rule, we obtain that $\mu x . \square x \rightarrow \gamma_{n}$ is provable in $\mathbf{K}^{\mu}$.

Theorem 3. $\mathbf{K}^{\mu}+\mu x . \square x$ is complete with respect to the class of finite tree Kripke models.

Proof. For any finite tree $\mathcal{M}$, we have $\mathcal{M} \Vdash \mathbf{K}^{\mu}$ and $\mathcal{M} \Vdash \mu x$. $\square x$. Thus, it is sufficient to show that if $\varphi$ is not provable in $\mathbf{K}^{\mu}+\mu x . \square x$, there exists a finite tree $N$ such that $N \nVdash \varphi$. Let $\varphi$ be such a formula. In particular, $\nvdash_{\mathbf{K}^{\mu}} \mu x . \square x \rightarrow \varphi$. By Theorem 2 we have $\mathcal{M}, w \nVdash \mu x . \square x \rightarrow \varphi$, for some generalized model $\mathcal{M}$ and some $w \in \mathcal{M}$.

Let $n$ be the rank of $\varphi$. By Theorem 2 and Proposition 5 , we get that $\mathcal{M}, w \Vdash$ $\mu x . \square x \rightarrow \gamma_{n}$. Since $\mathcal{M}, w \Vdash \mu x . \square x$, it follows that $\mathcal{M}, w \Vdash \gamma_{n}$. Therefore, there exists a finite tree $\mathcal{N}$ and some $v \in \mathcal{N}$ such that $(\mathcal{M}, w) \sim_{n}(\mathcal{N}, v)$. Since $\mathcal{M}, w \nVdash \varphi$, we have $\mathcal{N}, v \nVdash \varphi$.

As mentioned before, this result also follows from the completeness of $\mathbf{K}^{\mu}$ showed by Igor Walukiewicz in [11. We briefly explain how to derive Theorem 3 from the completeness of $\mathbf{K}^{\mu}$. Recall that in 11, Igor Walukiewicz showed that a sentence $\varphi$ is provable in $\mathbf{K}^{\mu}$ iff it is valid in all trees.

Suppose that a sentence $\varphi$ is not provable in $\mathbf{K}^{\mu}+\mu x . \square x$. In particular, the formula $\mu x . \square x \rightarrow \varphi$ is not provable in $\mathbf{K}^{\mu}$. It follows from the completeness of $\mathbf{K}^{\mu}$ that there is a model $\mathcal{M}=(W, R . V)$ and a point $w$ in $W$ such that $(W, R)$ is a tree and $\mu x . \square x \rightarrow \varphi$ is not true at $w$. We may assume that $w$ is the root of $(W, R)$.

Since $\mu x . \square x$ is true at $w$ and since $w$ is the root, it follows from Fact 1 that the tree $(W, R)$ is conversely well-founded. Let $n$ be the rank of $\varphi$. Now, if a point $v$ in $W$ has more than one successor of a given $n$-type $\theta$, we can pick one successor of $n$-type $\theta$ and delete all the other successors of $n$-type $\theta$. This would not modify the fact that $\varphi$ is not true at $w$. By doing this operation inductively and using the fact that $(W, R)$ is well-founded, we can prove that the tree $(W, R)$ may be assumed to be finite. Therefore, there is a finite tree $(W, R)$ in which $\varphi$ is not valid. 


\section{Adding Shallow Axioms to $\mathrm{K}^{\mu}+\mu x . \square x$}

By slightly modifying our method, it is also possible to prove that when we extend the logic $\mathbf{K}^{\mu}+\mu x$. $\square x$ with axioms that are shallow (defined below), we obtain complete axiomatizations for the corresponding class of finite trees.

Definition 13 ([10]). A formula is Prop-free if it is a sentence that does not contain any proposition letter. A formula is propositional if it is a sentence of the $\mu$-calculus that contains neither $\diamond$ nor $\mu$.

A formula is shallow if no occurrence of a proposition letter is in the scope of a fixpoint operator and each occurrence of a proposition letter is in the scope of at most one modality. In other words, the shallow formulas is the language defined by

$$
\varphi::=\psi|\diamond \psi| \varphi \vee \varphi \mid \neg \varphi,
$$

where $\psi$ is either a Prop-free formula or a propositional formula.

For example, $\diamond p \rightarrow \square p$ is a shallow formula. Other examples are formulas expressing that each point has at most two successors $(\diamond p \wedge \diamond(q \vee \neg p) \rightarrow \square(p \vee q))$, or that each point has at most one blind successor $(\diamond(p \wedge \square \perp) \wedge \square(\square \perp \rightarrow p))$.

The remaining of the section is devoted to the proof of the following completeness result. Recall that a formula $\varphi$ defines a class $\mathcal{C}$ of finite trees if $\mathcal{C}$ is exactly the class of trees which make $\varphi$ valid.

Theorem 4. Let $\varphi$ be a shallow formula. Then the logic $\mathbf{K}^{\mu}+\mu x . \square x+\varphi$ is complete with respect to the class of finite trees defined by $\varphi$.

In order to prove this result, as for the logic $\mathbf{K}^{\mu}+\mu x . \square x$, we first show that the logic is complete with respect to a class of generalized frames. To do so, we combine the completeness of the logic $\mathbf{K}^{\mu}$ with respect to the class of generalized frames together with a property of shallow formulas, which was proved in [10]. We first recall this property.

Definition 14. A generalized frame $\mathcal{F}=(W, R, \mathbb{A})$ is differentiated if for all $w, v \in W$ with $w \neq v$, there exists $A \in \mathbb{A}$ such that $w \in A$ and $v \notin A$.

A generalized model $\mathcal{F}=(W, R, \mathbb{A})$ is tight if for all $w, v \in W$ such that $(w, v) \notin R$, there exists $A \in \mathbb{A}$ such that $v \in A$ and for all $u \in A,(w, u) \notin R$.

A generalized frame is refined if it is differentiated and tight.

Definition 15. A formula $\varphi$ is persistent with respect to refined frames if for all refined frame $\mathcal{F}$ such that $\mathcal{F} \Vdash \varphi$, the formula $\varphi$ is valid on the underlying Kripke frame of $\mathcal{F}$.

Theorem 5 ([10]). Every shallow formula is persistent with respect to refined frames.

Theorem 6. Let $\varphi$ be a shallow formula. The logic $\mathbf{K}^{\mu}+\varphi$ is complete with respect to the class of generalized frames whose underlying Kripke frames make $\varphi$ valid. 
Proof. By Theorem 2, we know that the logic $\mathbf{K}^{\mu}+\varphi$ is complete with respect to a generalized model $\mathcal{N}=(W, R, V, \mathbb{A})$. Moreover, it follows from the proof of this theorem, that we may assume $\mathbb{A}$ to be the set $\left\{\llbracket \psi \rrbracket_{\mathcal{N}}: \psi\right.$ sentence $\}$. Therefore, it is enough to show that in the underlying Kripke frame $(W, R), \varphi$ is valid.

First we prove that $\varphi$ is valid in the generalized Kripke frame $(W, R, \mathbb{A})$. Let $V^{\prime}: \operatorname{Prop} \rightarrow \mathbb{A}$ be a valuation and let $\mathcal{N}^{\prime}$ be the generalized model $\left(W, R, V^{\prime}, \mathbb{A}\right)$. We have to show that $\mathcal{N} \Vdash \varphi$. It follows from the definition of $\mathbb{A}$ that for all proposition letters $p$, there is a formula $\varphi_{p}$ such that $V(p)=\llbracket \varphi_{p} \rrbracket_{\mathcal{N}}$. Now it is routine to show that

$$
\mathcal{N}^{\prime} \Vdash \varphi \quad \text { iff } \quad \mathcal{N} \Vdash \psi,
$$

where $\psi$ is a formula obtained by replacing each proposition letter $p$ occurring in $\varphi$ by the formula $\varphi_{p}$. Using Theorem 2, we obtain that $\mathcal{N} \Vdash \psi$ iff $\psi$ belongs to the logic $\mathbf{K}^{\mu}+\varphi$. Clearly, $\psi$ belongs to $\mathbf{K}^{\mu}+\varphi$ since this logic is closed under substitution and this finished the proof that $\varphi$ is valid in the generalized Kripke frame $(W, R, \mathbb{A})$.

Now, in Theorem 11, we could make the extra assumption that the model $\mathcal{M}=\left(W_{\mathcal{M}}, R_{\mathcal{M}}, V_{\mathcal{M}}\right)$ is such that the generalized frame $\left(W_{\mathcal{M}}, R_{\mathcal{M}}, \mathbb{A}_{\mathcal{M}}\right)$ is refined, where $\mathbb{A}_{\mathcal{M}}$ is the set $\left\{\llbracket \psi \rrbracket_{\mathcal{M}}: \psi\right.$ modal formula $\}$. This is a standard result and follows from the proof of Theorem 11. By looking at the proof of Theorem 2, we can see that this implies that the generalized frame $(W, R, \mathbb{A})$ (as defined in the first paragraph of this proof) is refined. Recall also that we proved that $\varphi$ is valid in this generalized frame. It follows from Theorem 5 that the formula $\varphi$ is valid in the $\operatorname{Kripke}$ frame $(W, R)$.

Definition 16. Let $\varphi$ be a formula and let $\mathcal{M}$ be a generalized model. A point $w \in \mathcal{M}$ is $n$-good for $\varphi$ if there exist a finite tree $\mathcal{G}$, a Kripke model $\mathcal{N}$ based on $\mathcal{G}$ such that $\mathcal{G} \Vdash \varphi$ and $(\mathcal{M}, w) \sim_{n}(\mathcal{N}, v)$, for some $v \in \mathcal{G}$.

Lemma 3. Let $\varphi$ be a formula and let $n$ be a natural number strictly greater than the rank of $\varphi$. There exists a formula $\delta_{n}$ of rank $n$ such that for all generalized models $\mathcal{M}$ and all $w \in \mathcal{M}$, we have

$$
\mathcal{M}, w \Vdash \gamma_{n} \quad \text { iff }(\mathcal{M}, w) \text { is } n \text {-good for } \varphi \text {. }
$$

Proof. Let $\gamma_{n}$ be the formula given by Lemma1. We can define $\delta_{n}$ as the formula $\gamma_{n} \wedge \mu x \cdot \varphi \wedge \square x$.

The proof of the next lemma is an easy adaptation of the proof of Lemma 2 Details are omitted.

Lemma 4. Let $\varphi$ be a shallow formula and let $n$ be a natural number strictly greater than the rank of $\varphi$. If $\delta_{n}$ is the formula given by Lemma 3, then $\vdash_{\mathbf{K}^{\mu}+\varphi}$ $\square \delta_{n} \rightarrow \delta_{n}$.

Proposition 6. Let $\varphi$ be a shallow formula and let $n$ be a natural number strictly greater than the rank of $\varphi$. If $\delta_{n}$ is the formula given by Lemma 3 , $\vdash_{\mathbf{K}^{\mu}} \mu x . \square x \rightarrow \gamma_{n}$. 
Proof. By Lemma 4 we know that $\square \delta_{n} \rightarrow \delta_{n}$ is provable in $\mathbf{K}^{\mu}+\varphi$. By the Fixpoint rule, we obtain that $\mu x . \square x \rightarrow \delta_{n}$ is provable in $\mathbf{K}^{\mu}+\varphi$.

Theorem 7. Let $\varphi$ be a shallow formula. The logic $\mathbf{K}^{\mu}+\mu x . \square x+\varphi$ is complete with respect to the class of finite tree defined by $\varphi$.

Proof. It is easy to see that every formula of the logic $\mathbf{K}^{\mu}+\mu x . \square x+\varphi$ is valid on all finite trees of the class defined by $\varphi$. Thus, it is sufficient to show that if $\psi$ is not provable in $\mathbf{K}^{\mu}+\mu x . \square x+\varphi$, there exists a finite tree $\mathcal{G}$ such that $\mathcal{G} \Vdash \varphi$ and $\mathcal{G} \nVdash \psi$. Let $\psi$ be such a formula. In particular, $\nvdash_{\mathbf{K}^{\mu}+\varphi} \mu x$. $\square x \rightarrow \psi$. By Theorem 6. there exist a generalized frame $\mathcal{F}$ and generalized model $\mathcal{M}$ based on $\mathcal{F}$ such that $\mathcal{F} \Vdash \varphi$ and $\mathcal{M}, w \nVdash \mu x . \square x \rightarrow \varphi$, for some $w \in \mathcal{F}$.

Let $n$ be a natural number strictly greater than the rank of $\varphi$ and greater or equal to the rank of $\psi$. By Theorem [6] and Proposition [6. we get that $\mathcal{M}, w \Vdash$ $\mu x . \square x \rightarrow \delta_{n}$. Since $\mathcal{M}, w \Vdash \mu x . \square x$, it follows that $\mathcal{M}, w \Vdash \delta_{n}$. Therefore, there exist a finite tree $\mathcal{G}$, a Kripke model $\mathcal{N}$ based on $\mathcal{G}$ and $v \in \mathcal{G}$ such that $\mathcal{G} \Vdash \varphi$ and $(\mathcal{M}, w) \sim_{n}(\mathcal{N}, v)$. Since $\mathcal{M}, w \nVdash \psi$, we have $\mathcal{N}, v \nVdash \psi$.

\section{Graded $\mu$-Calculus}

By adapting the definition of rank for the graded $\mu$-formulas, we can use the same proof to show that the graded $\mu$-calculus together with the axiom $\mu x . \square x$ is complete with respect to the class of finite trees. We start by recalling the definition of the graded $\mu$-calculus.

Definition 17. The formulas of the graded $\mu$-calculus are given by

$$
\varphi::=\top|p| x|\varphi \vee \varphi| \neg \varphi\left|\diamond^{k} \varphi\right| \mu x \cdot \varphi
$$

where $p$ ranges over the set Prop of proposition letters, $x$ ranges over the set Var of variables and $k$ is a natural number. In $\mu x . \varphi$, we require that the variable $x$ appears only under an even number of negations in $\varphi$. As before, we assume that $V$ ar is infinite and a graded modal formula is a formula which does not have any subformula of the form $\mu x . \varphi$.

For all natural numbers $k$, we let $\square^{k} \varphi$ be an abbreviation for $\neg \diamond^{k} \neg \varphi$. Moreover, for all $k>0$, we denote by $\diamond^{! k} \varphi$ the formula $\neg \diamond^{k} \varphi \wedge \diamond^{k-1} \varphi$. We also let $\diamond^{! 0} \varphi$ be the formula $\neg \diamond^{0} \varphi$.

Definition 18. Given a formula $\varphi$, a Kripke model $\mathcal{M}=(W, R, V)$ and an assignment $\tau: \operatorname{Var} \rightarrow \mathcal{P}(W)$, we define a subset $\llbracket \varphi \rrbracket_{\mathcal{M}, \tau}$ that is interpreted as the set of points at which $\varphi$ is true. The subset is defined by induction as before, with the extra requirement that

$$
\begin{aligned}
\llbracket \diamond^{k} \varphi \rrbracket_{\mathcal{M}, \tau}= & \left\{w \in W: \exists U \subseteq \llbracket \varphi \rrbracket_{\mathcal{M}, \tau} \cap\{u \in W: w R u\}\right. \\
& \text { s.t. } U \text { has at least } k+1 \text { elements }\} .
\end{aligned}
$$

The notions of truth and validity are defined as in Definition 2 
Definition 19. The axiomatization of the system $\mathbf{G K}^{\mu}$ consists of the following axioms and rules

$$
\begin{array}{lr}
\text { propositional tautologies, } & \\
\text { If } \vdash \varphi \rightarrow \psi \text { and } \vdash \varphi, \text { then } \vdash \psi & \text { (Modus ponens), } \\
\text { If } \vdash \varphi, \text { then } \vdash \varphi[p / \psi] & \text { (Substitution), } \\
\text { If } \vdash \varphi, \text { then } \vdash \square^{0} \varphi & \text { (Necessitation), } \\
\vdash \varphi[x / \mu x . \varphi] \rightarrow \mu x . \varphi & \text { (Fixpoint rule), } \\
\nabla^{k+1} p \rightarrow \diamond^{k} p & \text { (axiom G1), } \\
\square^{0}(p \rightarrow q) \rightarrow\left(\diamond^{n} p \rightarrow \diamond^{n} q\right) & \text { (axiom G2), } \\
\diamond^{!}(p \wedge q) \rightarrow\left(\left(\diamond^{!} p \wedge \diamond^{! l} q\right) \rightarrow \diamond^{! k+l}(p \vee q)\right) & \text { (axiom G3), } \\
\vdash \varphi[x / \mu x . \varphi] \rightarrow \mu x . \varphi & \text { (Fixpoint axiom), } \\
\text { If } \vdash \varphi[x / \psi] \rightarrow \psi, \text { then } \vdash \mu x . \varphi \rightarrow \psi & \text { (Fixpoint rule), }
\end{array}
$$

(Substitution),

(Necessitation),

(Fixpoint rule),

(axiom $G 1)$,

(axiom $G 2$ ),

(axiom G3),

(Fixpoint axiom),

(Fixpoint rule),

where $x$ is not a bound variable of $\varphi$ and no free variable of $\psi$ is bound in $\varphi$.

The logic GK is the smallest set of formulas which contains the propositional tautologies, the axioms $G 1, G 2$ and $G 3$ and is closed under the Substitution, the Modus ponens and the Necessitation rules.

Theorem 8 ([4]). The logic GK is complete with respect to a single model. That is, there is a Kripke model $\mathcal{M}$ such that a graded modal formula is provable in $\mathbf{G K}$ iff it is true in $\mathcal{M}$.

Theorem 9. The logic $\mathbf{G K}^{\mu}+\mu x \cdot \square^{0} x$ is complete with respect to the class of finite trees. That is, a graded $\mu$-formula is provable in $\mathbf{G K}^{\mu}+\mu x . \square^{0} x$ iff it is valid in all finite trees.

Proof (sketch). The structure of the proof is the same as the one for the proof of Theorem 3 . So first, we need to define a notion of rank for graded $\mu$-formulas. As before, we start by defining the closure and the depth of a formula. The closure of graded formula is defined as in Definition 6, except that we replace $\diamond$ by $\diamond^{k}$. The depth of a graded $\mu$-formula is defined by induction as follows

$$
\begin{array}{l|l}
d(\top)=d(p)=d(x)=0, & d(\varphi \vee \psi)=\max \{d(\varphi), d(\psi)\}, \\
d(\neg \varphi)=d(\varphi), & d\left(\diamond^{k} \varphi\right)=d(\varphi)+k+1, \\
d(\mu x . \varphi)=d(\varphi)+1 . &
\end{array}
$$

Finally, we can define the rank of a graded $\mu$-formula as in Definition 8 .

The second step is to prove completeness of $\mathbf{G K}^{\mu}$ with respect to the class of generalized frames. Using Theorem 8, it is possible to show this by using a proof that is completely similar to the proof of Theorem 2. We do not give details.

We can do so easily this step because we gave a proof of Theorem 2 which uses directly the completeness result for the modal case, instead of adapting the canonical model construction for the modal case to the $\mu$-calculus. Indeed, the canonical model construction for graded modal logic is rather difficult and it would not be immediate to adapt it when fixpoints are added.

The last step is to show the completeness of $\mathbf{G K}^{\mu}+\mu x \cdot \square^{0} x$ with respect to the class of finite trees. This is done by extending all the notions and results of section 4 to the setting of the graded $\mu$-calculus. It is immediate how to proceed. 


\section{Further Work}

We believe that this method could be adapted to other cases. In the last section, we considered the fixpoint version of graded modal logic. Graded modal logic is an extension of modal logic with some sort of counting. We could look at fixpoint versions of modal logic extended with richer form of counting. An example would be Presburger modal logic (see, e.g., 2]). Finally, we would like to mention that Stéphane Demri (p.c.) raised the question to which class of coalgebras this proof could be adapted.

\section{References}

1. Bojańczyk, M.: Effective characterizations of tree logics. In: PODS 2008: Proceedings of the twenty-seventh ACM SIGMOD-SIGACT-SIGART symposium on Principles of database systems, pp. 53-66. ACM, New York (2008)

2. Demri, S., Lugiez, D.: Presburger modal logic is only PSPACE-complete. In: Furbach, U., Shankar, N. (eds.) IJCAR 2006. LNCS (LNAI), vol. 4130, pp. 541-556. Springer, Heidelberg (2006)

3. Doets, K.: Monadic $\Pi_{1}^{1}$-theories of $\Pi_{1}^{1}$-properties. Notre Dame Journal of Formal Logic 30(2) (1989)

4. Fattorosi-Barnaba, M., Cerrato, C.: Graded modalities I. Studia Logica, 47 (1988)

5. Fischer, M., Ladner, R.: Propositional dynamic logic of regular programs. Journal of Computer and System Sciences 18(2) (1979)

6. Gheerbrant, A., ten Cate, B.: Complete axiomatizations of MSO, FO(TC1) and FO(LFP1) on finite trees. In: LFCS (2009)

7. Kozen, D.: Results on the propositional $\mu$-calculus. In: Lee, I., Smolka, S.A. (eds.) CONCUR 1995. LNCS, vol. 962, Springer, Heidelberg (1995)

8. Libkin, L.: Logics for unranked trees: An overview. Logical Methods in Computer Science 2(3) (2006)

9. de Rijke, M., Blackburn, P., Venema, Y.: Modal Logic. Cambridge University Press (1991)

10. ten Cate, B.: Model theory for extended modal languages. PhD thesis, University of Amsterdam, ILLC Dissertation Series DS-2005-01 (2005)

11. Walukiewicz, I.: A note on the completeness of Kozen's axiomatization of the propositional $\mu$-calculus. The Bulletin of Symbolic Logic 2(3) (1996) 